\title{
ASYMMETRY OF RADIAL HEAT PULSE PROPAGATION AT TEXTOR
}

\author{
A. KRÄMER-FLECKEN, A. ROGISTER, M.Z. TOKAR'*, \\ G. WAIDMANN, G.H. WOLF \\ Institut für Plasmaphysik, \\ Forschungszentrum Jülich, \\ Association Euratom/KFA, \\ Jülich, Germany
}

\begin{abstract}
Electron heat pulse propagation following sawtooth collapse has been simultaneously observed along two opposite radial directions. For a given flux surface, the arrival of the heat pulse at the high field side (HFS) is delayed by up to $1 \mathrm{~ms}$ as compared with the arrival at the low field side (LFS). The observed delay agrees with an estimate of the time required for parallel electron heat transport along the magnetic field lines connecting the corresponding LFS and HFS isobars. This suggests that the primary radial heat transport occurs on the LFS, whereas the HFS is fed subsequently from the LFS by temperature equilibration along the connecting magnetic field lines.
\end{abstract}

\section{INTRODUCTION}

It is usually assumed that electron heat transport is governed by a diffusive process across the entire magnetic surface, and consequently that the electron heat diffusivity $\chi_{\mathrm{e}}(r)$ is a useful characteristic quantity to describe this process. Experimentally, $\chi_{\mathrm{e}}(r)$ is commonly derived from the (quasi-)stationary energy balance [1]. Complementing this standard procedure, perturbation methods have been applied to calculate the $\chi_{\mathrm{e}}$ values from the radial propagation of transients on the electron temperature $T_{\mathrm{e}}$ and electron density $n_{\mathrm{e}}$ profiles [2, 3]. An intrinsic source of such transients is the sawtooth collapse occurring in tokamaks under many discharge conditions.

Previous analyses of the heat pulse propagation following the sawtooth collapse have been based on observations along one radial direction only, except for the most recent analysis [4]. They have addressed the question of whether, and to what extent, the $\chi_{\mathrm{e}}$ values derived from heat pulse propagation differ from those obtained from the stationary power balance.

The present paper reports on the simultaneous observation of heat pulse propagation along two radial directions in the equatorial plane, that is along both the high field side (HFS) and the low field side (LFS). The measured delay between the arrival of the heat pulses on the same magnetic surface leads to the conclusion

* Permanent affiliation: Institute for High Temperatures, Moscow, Russia. that the observed pattern, initiated by an asymmetric sawtooth crash, can only be explained by the combined action of parallel and perpendicular transport.

\section{THE EXPERIMENTAL SET-UP}

At TEXTOR $\left(B_{\mathrm{T}}=2.25 \mathrm{~T}\right.$ at $R_{0}=1.75 \mathrm{~m}$, $a=0.46 \mathrm{~m}$ ), a heterodyne ECE diagnostic [5] had been installed to measure the electron temperature on the equatorial plane at 10 different radial positions. The radiometer frequencies are radially placed from 105 to $145 \mathrm{GHz}$, in steps of $5 \mathrm{GHz}$. The spatial resolution of each system is $\Delta R= \pm 0.7 \mathrm{~cm}$ and the data sampling rate is $10 \mathrm{kHz}$. On both the LFS and the HFS a tunable radiometer is available, covering a frequency range of $137 \leq f \leq 150 \mathrm{GHz}$ on the HFS and $102 \leq f \leq 113 \mathrm{GHz}$ on the LFS. To measure the electron temperature dynamics in steps of $1 \mathrm{GHz}$, 15 reproducible discharges were necessary.

A multichannel $\mathrm{HCN}$ interferometer [6] positioned $180^{\circ}$ toroidally apart from the ECE system, and looking vertically through the plasma, yielded information on the electron density profiles and on the temporal development of the local electron density during the sawtooth collapse. The spatial resolution of each interferometer channel yields $\Delta r=2 \mathrm{~cm}$. Three interferometer channels on the HFS and four interferometer channels on the LFS are placed in the region that is covered by the tunable radiometers. 
The radial temperature and density developments were measured for two series of ohmically heated plasmas with the following plasma parameters: $I_{\mathrm{p}}=350 \mathrm{kA}$, $\bar{n}_{\mathrm{e}}=2.1 \times 10^{13} \mathrm{~cm}^{-3}, \beta_{\mathrm{pol}}=0.32$ and $B_{\mathrm{T}}=2.25$, respectively, with $B_{\mathrm{T}}=2.22 \mathrm{~T}$ on the geometrical axis. The $Z_{\text {eff }}$ values were measured to be $Z_{\text {eff }}=1.5$ (if carbon is the major impurity) for the first series $\left(B_{\mathrm{T}}=2.25 \mathrm{~T}\right)$ and $Z_{\mathrm{eff}}=2.0$ for the second one $\left(B_{\mathrm{T}}=2.22 \mathrm{~T}\right)$. In order to increase the statistical accuracy, a time interval from $0.5 \leq t \leq 1.2 \mathrm{~s}$ was analysed containing around 40 individual sawtooth events for each discharge.

\section{EXPERIMENTAL RESULTS}

\subsection{Heat pulse propagation time}

The heat pulse propagation analysis is confined to an area outside the sawtooth inversion radius. After a sawtooth crash in the plasma centre, the heat pulse propagation time $t_{\mathrm{p}}$ at a certain radial position $R_{0} \pm r$ is defined as the time interval after which the temperature maximum $\Delta T_{\mathrm{e}}$ of the heat pulse has been reached. The maximum in the $T_{\mathrm{e}}$ variation deduced from the central ECE channel during the sawtooth crash defines the starting time, $t=0$.

The raw data were superimposed with statistical noise and contributions from plasma fluctuations, making it difficult to estimate $t_{\mathrm{p}}$. In the analysed region, from $0.5-1.2 \mathrm{~s}$, the sawtooth period was stable to within $5 \%$. After a fast Fourier transformation of the raw data, a band pass filter covering the first four harmonics of the sawtooth period was used to reduce the high frequency contributions to the frequency spectrum. The width of each frequency band was $\Delta \omega= \pm 8 \mathrm{~Hz}$ at the $n$th harmonic. From the first four harmonics of the sawtooth period, the heat pulse propagation time $t_{\mathrm{p}}$ was obtained for the different radial positions. The radial positions were estimated from the superposition of the toroidal and poloidal magnetic fields. The poloidal magnetic field has been calculated assuming a parabolic current density profile with $j_{0}=2 \mathrm{MA} / \mathrm{m}^{2}$ [7].

For both discharge series, the general behaviours of the heat pulse propagation on the HFS and on the LFS were found to be similar. As a general feature, the velocity of the radial heat pulse propagation decreased with increasing radial distance from the origin of the collapse. From the radial dependence of the heat pulse propagation time, we deduced a velocity of $v=35 \mathrm{~m} / \mathrm{s}$ at $\left|R-R_{0}\right| / a=0.55$ on the HFS and of $v=34 \mathrm{~m} / \mathrm{s}$ at $\left|R-R_{0}\right| / a=0.84$ on the LFS for $B_{\mathrm{T}}=2.25 \mathrm{~T}$. For the discharge series with $B_{\mathrm{T}}=2.22 \mathrm{~T}$, the same analysis yields velocities of $v=33 \mathrm{~m} / \mathrm{s}$ at $\left|R-R_{0}\right| / a=0.63$ on the HFS and $v=29 \mathrm{~m} / \mathrm{s}$ at $\left|R-R_{0}\right| / a=0.75$ on the LFS.

\subsection{Evolution of $T_{\mathrm{e}}$ profiles during sawtooth crash}

The electron temperature profiles were obtained from coherently averaged ECE data. During the time interval investigated, around 40 sawtooth periods were overlaid, taking the sawtooth crash in the plasma centre (defined by the largest temperature variation), as the starting signal.

For both discharge series the inversion radius, deduced from the $T_{\mathrm{e}}$ profiles, was about $r_{\mathrm{INV}} \approx 10 \mathrm{~cm}$. Precursor activity just before the sawtooth crash occurred with a frequency of about $3.3 \mathrm{kHz}$, corresponding to an oscillation period of $\tau=300 \mu \mathrm{s}$.

At the toroidal position of the ECE diagnostic, the starting position of the sawtooth crash was always localized on the LFS (Fig. 1). The onset of the sawtooth crash on the LFS at $t=200 \mu \mathrm{s}$ is clearly seen. Curve 3 displays a temperature increase outside the inversion radius. No indication of a temperature increase on the HFS was found for this time. The sawtooth crash has a fixed correlation to the precursor oscillations. The starting point of the sawtooth crash

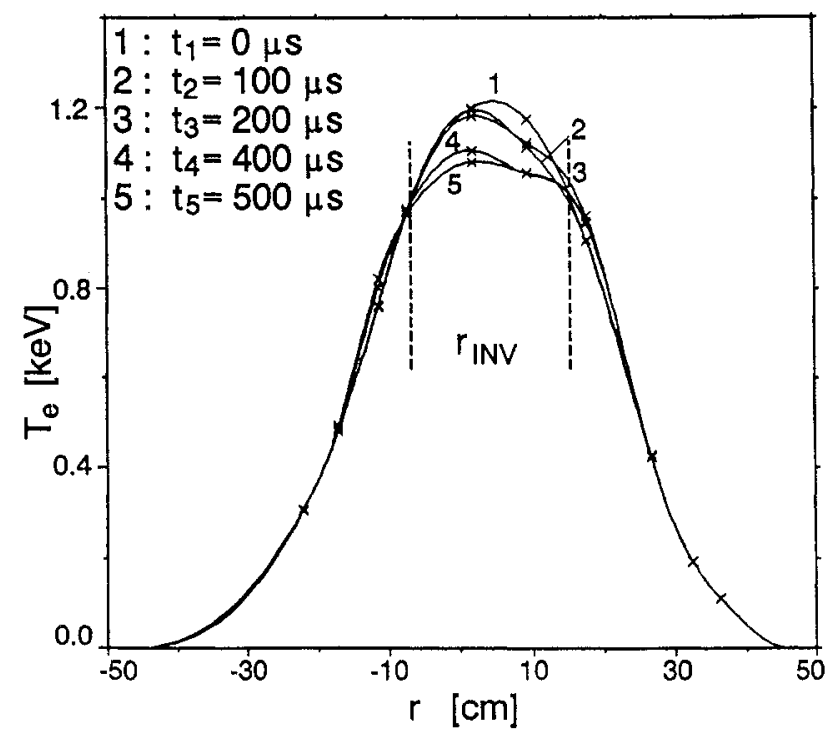

FIG. 1. Development of the $\mathrm{T}_{e}$ profile during the sawtooth crash. The profile is obtained from coherently averaged data. The consecutive profiles show the occurrence of inverted sawteeth on the LFS (curve 3). At this time, no evidence for a region of inverted sawteeth on the HFS was found. Curve 2 displays a temperature decrease on the LFS due to mode activity. 


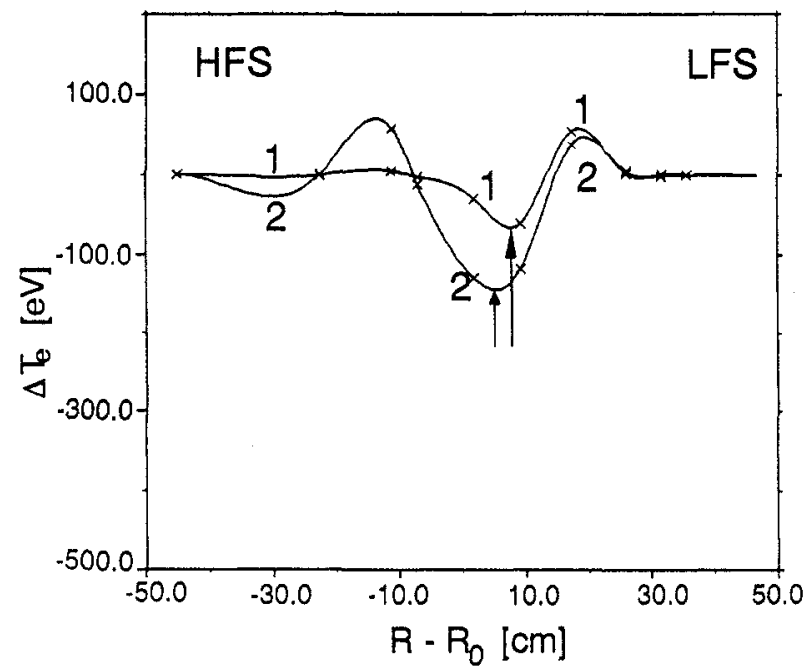

FIG. 2. Temperature difference, $\Delta \mathrm{T}_{e}(\mathrm{r})$, deduced from two $\mathrm{T}_{e}$ profiles $\left(\mathrm{t}_{3}-\mathrm{t}_{l}\right.$ from Fig. 1) at the beginning of the sawtooth crash, $\Delta \mathrm{t}=200 \mu \mathrm{s}$ (curve 1). Curve 2 displays $\Delta \mathrm{T}_{e}(\mathrm{r})$ for the subtraction of $a \mathrm{~T}_{e}$ profile after the sawtooth crash from one at the onset of the crash $\left(\Delta \mathrm{t}=400 \mu \mathrm{s} ; \mathrm{t}_{5}-\mathrm{t}_{1}\right.$ from Fig. 1). The arrows mark the position of the crash centre.

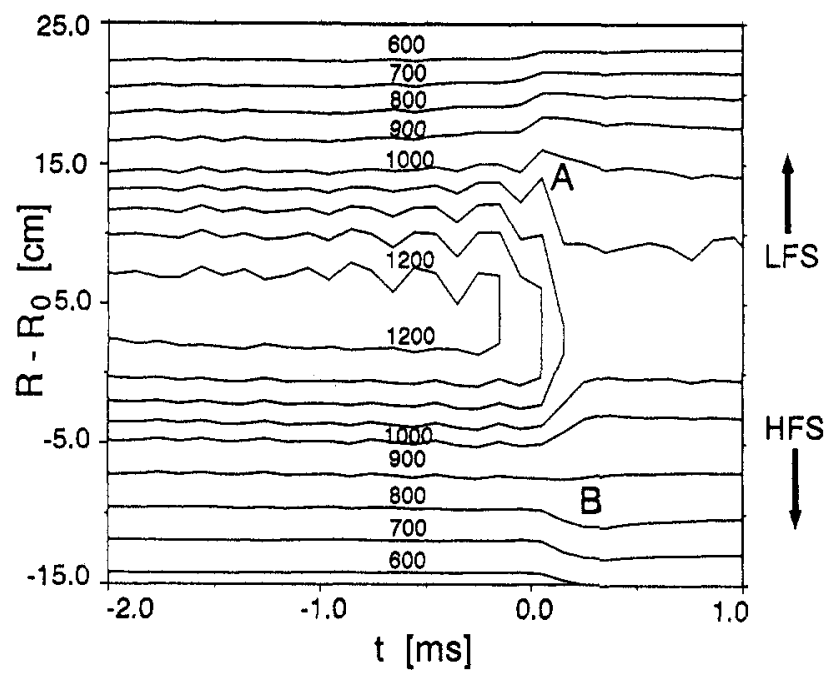

FIG. 3. A $\mathrm{T}_{e}$ contour plot displaying the sawtooth crash, the release of the plasma on the LFS and the delay between the occurrence of the inverted sawteeth on the LFS and the HFS. The isotherms up to $\mathrm{T}_{e}=1000 \mathrm{eV}$ are plotted in steps of $100 \mathrm{eV}$. For $\mathrm{T}_{e}>1000 \mathrm{eV}$ they are plotted in steps of $50 \mathrm{eV}$. ' $A$ ' indicates the spike on the isotherms on the LFS and ' $B$ ' indicates the first occurrence of an inverted sawtooth on the HFS.

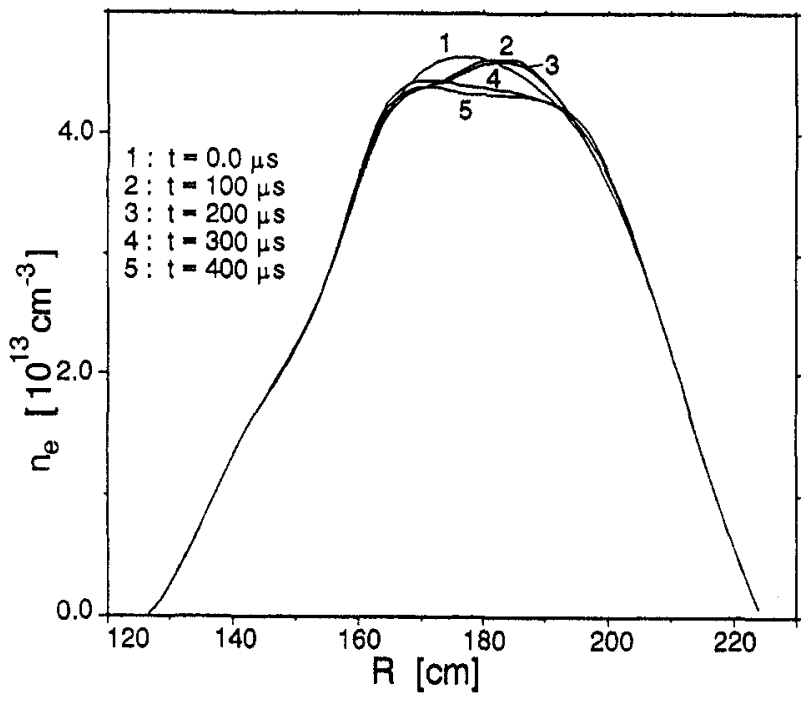

FIG. 4. Profiles of $\mathrm{n}_{e}$ displaying the asymmetry and the development of the sawtooth crash. The HCN interferometer is separated $180^{\circ}$ toroidally from the ECE diagnostic. From the HCN data the sawtooth crash has its onset on the HFS (curve 3).

inside the inversion radius is always located in the minimum of the precursor oscillation on the LFS. From the LFS the crash moved within about $200 \mu \mathrm{s}$ towards the HFS, that is during a time that corresponds to half of the precursor period. Looking for temperature changes, by subtracting subsequent $T_{\mathrm{e}}$ profiles during the onset phase of the sawtooth crash from each other, the LFS exhibits a radial region where the temperature increases, that is a region of inverted sawteeth, whereas no indications for such an inverted region were found on the HFS (Fig. 2). However, subtracting the $T_{\mathrm{e}}$ profiles just before the sawtooth crash from those taken $400 \mu$ s later shows a region of inverted sawteeth on both the HFS and the LFS. Moreover, comparing the curves shows that the (radial) position of the maximum temperature amplitude in the centre has moved slightly towards the HFS, as indicated by the arrows in Fig. 2.

Both the sawtooth crash dynamics and the early state of the heat pulse development also become visible from a $T_{\mathrm{e}}$ contour plot of the averaged ECE data covering the vicinity of the sawtooth crash (Fig. 3). A pronounced spike on the LFS isotherm develops there during the sawtooth crash (A), indicating that the plasma outflow from a hot spot was localized on the LFS. This spike disturbs the isotherms up to $r \approx 24 \mathrm{~cm}$ on the LFS, whereas on the HFS no indication of an equivalent process was found but rather a common shift, of the isotherms with $T_{\mathrm{e}} \geq 900 \mathrm{eV}$, towards the LFS. 


\section{KRÄMER-FLECKEN et al.}

The first indications for the occurrence of the heat pulse on the HFS were observed on the $T_{\mathrm{e}}=850 \mathrm{eV}$ isotherm (B) about $\Delta t \approx 200 \mu \mathrm{s}$ after the sawtooth crash. If the hot spot is the subject of a poloidal rotation, that is one of an $m=1, n=1$ mode structure and by accounting for the precursor period of $300 \mu \mathrm{s}$, an outflow of hot plasma on the HFS should occur about $200 \mu \mathrm{s}$ after the onset of the sawtooth crash on the LFS. However, since no indication of such an outflow of the hot plasma towards the HFS could be found, the release of the hot plasma is assumed to occur only on the LFS.

The opposite behaviour was found when analysing the $n_{\mathrm{e}}$ profiles, measured by the $\mathrm{HCN}$ interferometer, separated $180^{\circ}$ toroidally from the $T_{\mathrm{e}}$ diagnostic, displaying the beginning of the sawtooth crash on the HFS (Fig. 4). The same correlation between the precursor activity and the sawtooth crash was also found in the $n_{\mathrm{e}}$ profile [8]. Furthermore, the combined results of both diagnostics support the assumption of an $m=1, n=1$ mode structure existing before the sawtooth crash.

From the asymmetry in the sawtooth crash, an initial time delay between the HFS and the LFS is expected. Comparing the inverted $T_{\mathrm{e}}$ sawtooth signal outside the inversion radius, we found a time offset of $\Delta t \sim 300 \mu \mathrm{s}$ between the radiometer EC4 on the HFS located at $R-R_{0}=-18.0 \mathrm{~cm}$ and the radiometer EC1 at the radial position $R-R_{0}=27.1 \mathrm{~cm}$ on the LFS (Fig. 5(a)). Within $\Delta r=5 \mathrm{~mm}$, both positions correspond to the same original isobar.

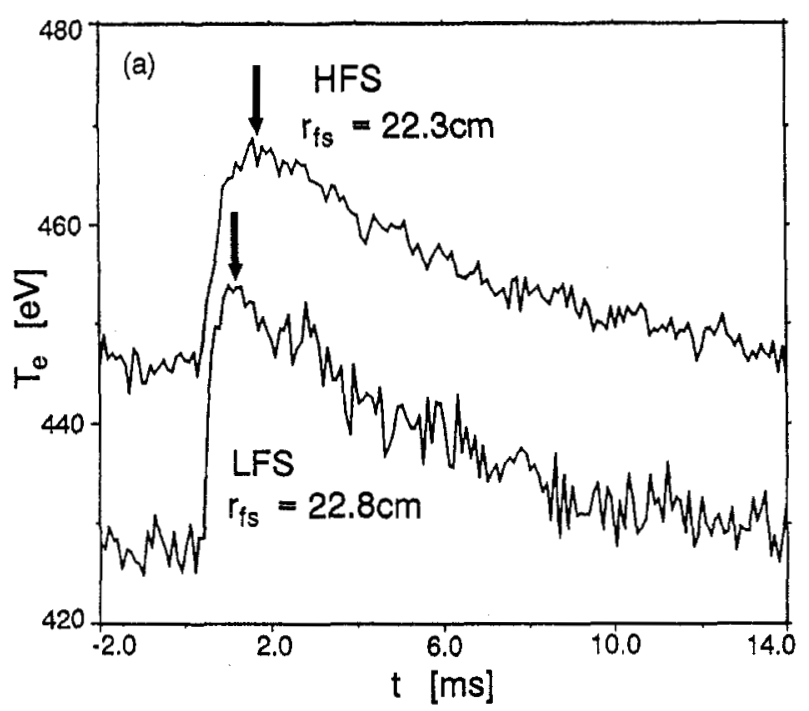

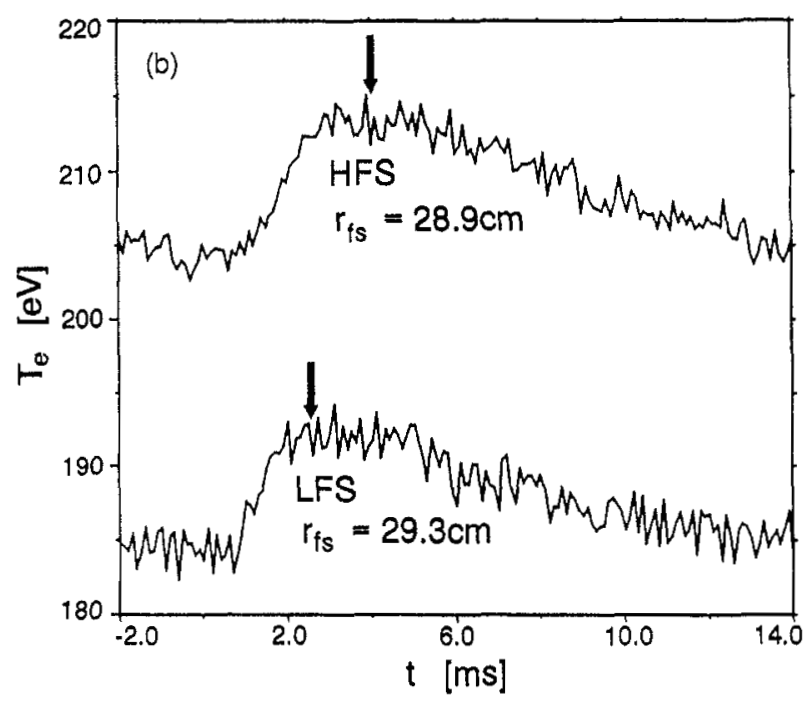

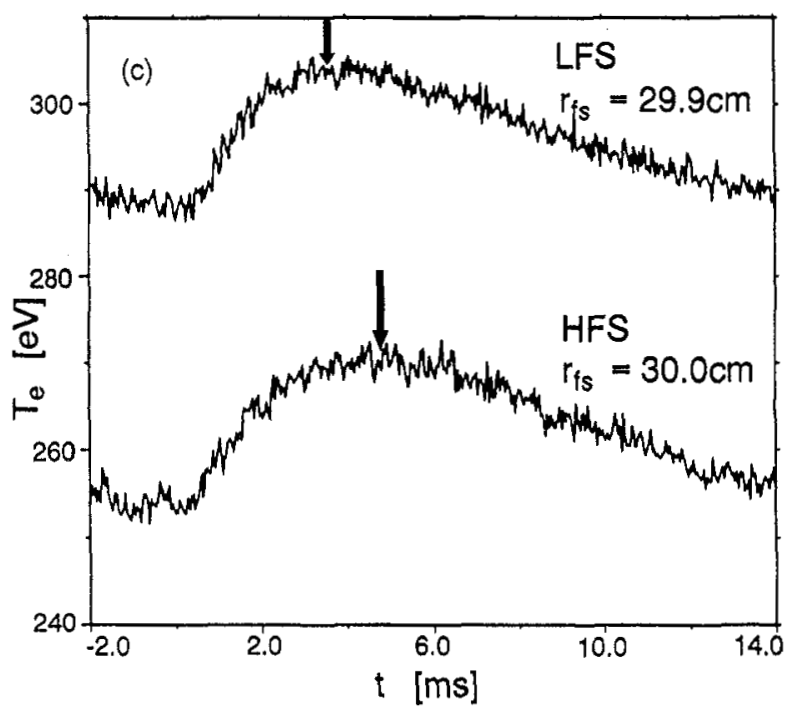

FIG. 5. Comparison of the inverted sawteeth for three different isobars for both the LFS and the HFS. Parts (a) and (b) are deduced from the experiment where the radiometer frequency was varied. Part (c) shows an example from the experiment where the horizontal plasma position was changed. The time offsets between the LFS and the HFS are clearly seen. Arrows indicate the position of $t_{p}$.

\section{INTERPRETATION OF THE DATA}

\subsection{Heat pulse propagation on isobars}

For a comparison of the heat pulse propagation on the LFS and on the HFS, a transformation into the coordinate system of the flux surfaces, which is assumed to be characterized by isotherms or isobars, is necessary. The existing deviations between isotherms and lines of 

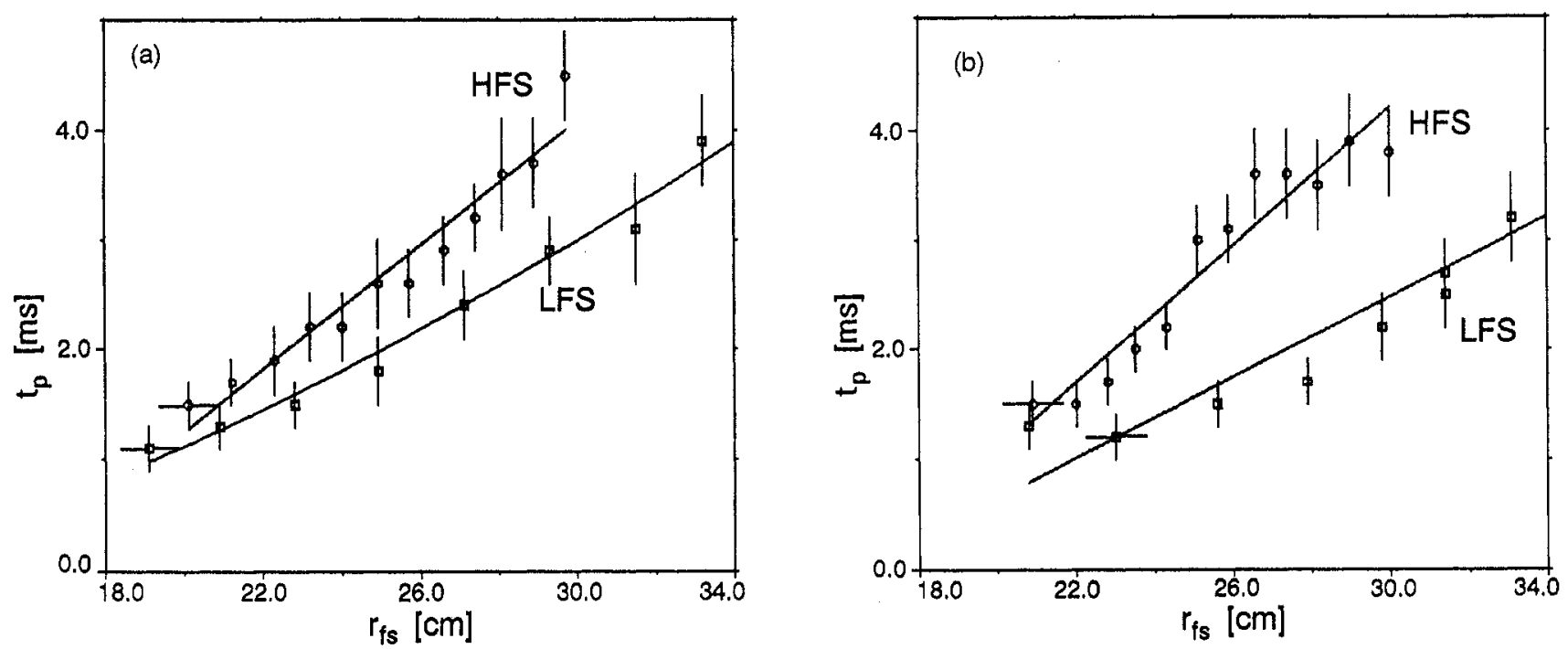

FIG. 6. Heat pulse propagation time versus isobar radius for the two series. The spatial resolution is shown for the first measured data point. Both series display a time offset between the LFS and the HFS, and a much faster heat pulse propagation on the LFS. The plasma parameters are: (a) $\mathrm{I}_{p}=350 \mathrm{kA}, \mathrm{B}_{T}=2.25 \mathrm{~T}, \overline{\mathrm{n}}_{e}=2.1 \times 10^{13} \mathrm{~cm}^{-3}, \mathrm{Z}_{e f f}=1.5 ;(b) I_{p}=350 \mathrm{kA}, \mathrm{B}_{T}=2.22 T$, $\overline{\mathrm{n}}_{e}=2.1 \times 10^{13} \mathrm{~cm}^{-3}, \mathrm{Z}_{\text {eff }}=2.0$.

constant flux yield $\Delta r= \pm 2 \mathrm{~mm}$. In order to obtain the corresponding pressure $p_{\mathrm{e}}$ profiles, the $\mathrm{HCN}$ data were also coherently averaged, using the sawtooth crash in the plasma centre as the trigger signal. The resulting density profile, averaged over the precursor activity before the crash, was multiplied by the temperature profile (taken in the same time interval as the density profile). This procedure yields the 'unperturbed' $p_{\mathrm{e}}$ profile and its displacement with respect to the geometrical axis, $R_{0}=1.75 \mathrm{~m}$. To make sure that the influence of the sawtooth crash on the reference isobars thus obtained is small, the $p_{\mathrm{e}}$ profile after the sawtooth crash was also calculated: the isobar radii and the displacement from the geometrical axis deduced from these two profiles coincide in the relevant radial range of flux surface radius of $16 \leq r_{\mathrm{fs}} \leq 35 \mathrm{~cm}$ (corresponding to $-14.5 \leq r_{\mathrm{HFS}} \leq-29.5 \mathrm{~cm}$ and $20.0 \leq r_{\mathrm{LFS}} \leq 42 \mathrm{~cm}$ ) within a margin of $0.1 \leq \Delta r \leq 0.3 \mathrm{~cm}$. Since these deviations are less than the spatial resolution of the ECE diagnostic, averaged profiles (covering the whole time interval analysed) can also be used. Plotting the heat pulse propagation time as a function of the isobar radius (Fig. 6) exhibits a distinct difference in the propagation time between the HFS and the LFS. In both series, at the toroidal position of the ECE diagnostic, the heat pulse propagation is significantly faster on the LFS than on the HFS. Already during the initial development of the heat pulse occurring just outside the inversion radius such a temporal asymmetry was found, which is the result of the preceding process, as described in Section 3.2 and in Figs 1 and 2. In Fig. 6(a), the error bars of the heat pulse propagation time overlap in some cases, but a systematic time difference $\Delta t_{\mathrm{p}}$ between the LFS and the HFS is visible, which increases with the isobar radius. In Fig. 6(b), this time difference becomes even more pronounced. For an isobar radius of $30 \mathrm{~cm}, \Delta t_{\mathrm{p}}$ reached values of up to $\Delta t_{\mathrm{p}} \approx 1 \mathrm{~ms}$.

The observed deviations in the measured $t_{\mathrm{p}}$ values fall outside the uncertainty between the lines of constant flux and isotherms, respectively isobars. In fact, to obtain fictitiously an equal propagation time (on isotherms) along both directions, a difference of $100 \mathrm{eV}$ between the HFS and the LFS was found for $t_{\mathrm{p}}=2.9 \mathrm{~ms}$. This temperature difference would correspond to a radial shift between the isotherms and the lines of constant magnetic flux of more than $2 \mathrm{~cm}$, which is outside the uncertainty limits (the spatial resolution of the ECE radiometer, as well as the difference between the isotherms and the flux surfaces). These results remain essentially the same if, instead of the $p_{\mathrm{e}}$ profile, the $n_{\mathrm{e}}$ profile or $T_{\mathrm{e}}$ profile was taken as the reference frame for the estimation of the displacement from the geometrical axis as well as for the radius of those lines having equal $n_{\mathrm{e}}$ or $T_{\mathrm{e}}$. For circular surface elements (in a poloidal section) having constant $n_{\mathrm{e}}$ or $T_{\mathrm{e}}$, we calculated, with measured $T_{\mathrm{e}}$ or $n_{\mathrm{e}}$ profiles, respectively, a similar development of the radial displacement 


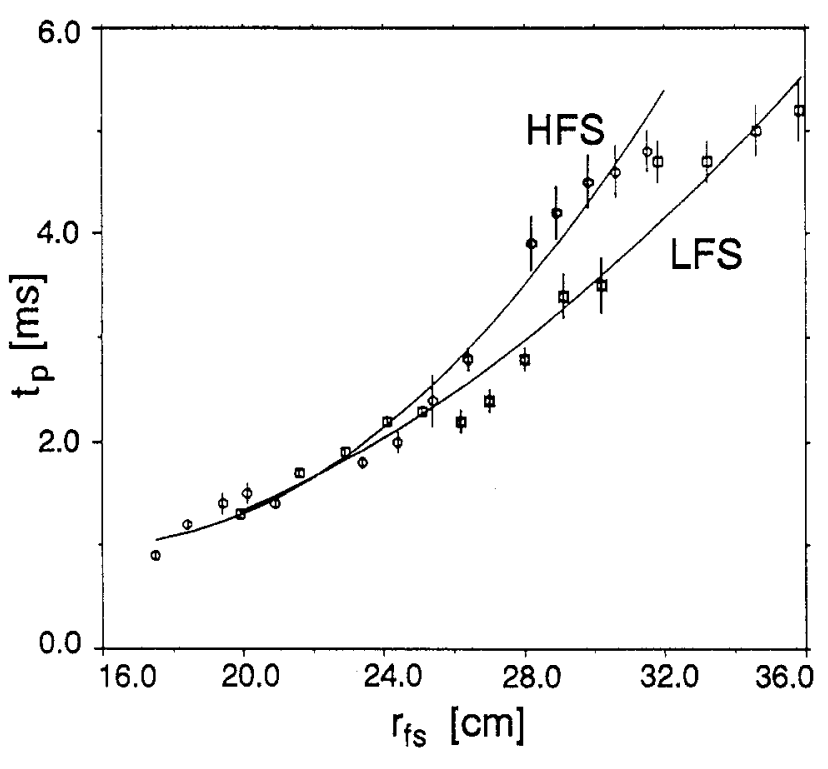

FIG. 7. Heat pulse propagation time versus isobar radius (observed by shifting the horizontal plasma position).

(the sum of the Shafranov shift and the horizontal plasma position).

Furthermore, we performed a discharge series $\left(I_{\mathrm{p}}=350 \mathrm{kA}, B_{\mathrm{T}}=2.25 \mathrm{~T}\right.$ and $\left.\bar{n}_{\mathrm{e}}=2.5 \times 10^{13} \mathrm{~m}^{-3}\right)$ where the horizontal position of the density peak was changed from $R-R_{0}=-0.7 \mathrm{~cm}$ to $R-R_{0}=8.0 \mathrm{~cm}$, with fixed radiometer frequency settings. Owing to the shot-by-shot variation of the plasma position relative to the radial ECE position given by the toroidal magnetic field, different radial positions in the co-ordinate system of the plasma become accessible. Also in this case, the analysis showed a much faster radial heat pulse propagation on the LFS than on the HFS (Fig. 7).

One possible reason for the different development of the $t_{\mathrm{p}}$ values shown in Figs 6 and 7 may be caused by the differences in the observed $T_{\mathrm{e}}$ profile (as a result of different discharge conditions).

In view of the observed difference in the heat pulse propagation between the HFS and the LFS, the question arises of whether there is also some difference in the amplitudes of the inverted sawteeth on the HFS and the LFS for corresponding isobar radii. The development of the inverted sawtooth amplitude versus isobar radius is shown in Fig. 8. For $r_{\mathrm{fs}} \leq 24 \mathrm{~cm}$, a strong difference was indeed found between the HFS and the LFS. At the beginning of the observation interval, the measured amplitude on the LFS is nearly twice as high as that on the HFS. For $r_{\mathrm{fs}} \geq 25 \mathrm{~cm}$, however, the measurement of the amplitudes becomes perturbed by noise and the difference in amplitudes between the LFS and the HFS diminished. Furthermore, the density per- turbation caused by the sawtooth crash had vanished at $r_{\mathrm{fs}} \approx 25 \mathrm{~cm}$. Neither observation, either that of the asymmetric heat pulse propagation or that of the asymmetric development of the inverted sawtooth amplitude, appears to be compatible with the concept of a predominantly global diffusive process across the entire magnetic surface. Instead, they indicate the dominance of a rather localized primary radial heat transport mechanism at the LFS, whereas in a subsequent process the heat is transported from the LFS to the HFS along the connecting field lines via parallel heat conduction. The isotherm pattern, as shown in Fig. 2, reveals this impression in a suggestive manner. This is in analogy to a flow of water that is forced to pulsate in an axisymmetric cascade fountain that consists of superimposed concentric circular basins that are, however, not properly aligned in the horizontal plane but slightly tilted to one side.

Considering the whole toroidal circumference, we assume that the radial heat pulse is launched from a helical starting position. This is in agreement with the observations from the $\mathrm{HCN}$ interferometer [8].

\subsection{Estimate of parallel heat propagation}

In order to test the consistency of the proposed transport model, the electron heat transfer time between the LFS and the HFS along the connecting magnetic field lines has to be estimated. Two approximations to this problem were performed.

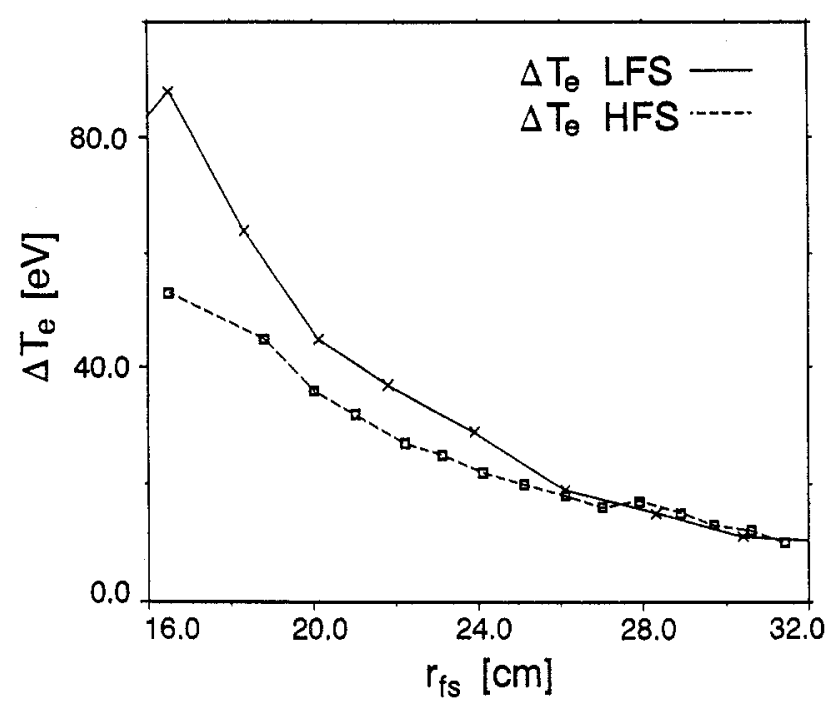

FIG. 8. Asymmetric development of the inverted sawtooth amplitude plotted versus isobar radius. The amplitudes of the LFS and HFS become equal for a radius of $\mathrm{r}_{f_{s}} \geq 25 \mathrm{~cm}$. 
In the first approach, we analyse the ratio between the connection length $L_{\mathrm{c}}$ for the transport along the magnetic field $\boldsymbol{B}$ (co-ordinate $l$ ) between the LFS and the HFS, and the path length $\lambda_{C}$ between Coulomb collisions. Considering a magnetic surface with a value of $q \approx 2$, we obtain $L_{\mathrm{c}}=\pi q R_{0}$, that is (for $\left.R_{0}=1.75 \mathrm{~m}\right) L_{\mathrm{c}} \approx 11 \mathrm{~m}$. By comparison, an estimate of $\lambda_{\mathrm{C}} \approx 10^{10} T_{\mathrm{e}}^{2} / n_{\mathrm{e}}\left(\mathrm{m}, \mathrm{eV}, \mathrm{cm}^{-3}\right)$ yields values in the range of $8-180 \mathrm{~m}$, since $100 \leq T_{\mathrm{e}} \leq 650 \mathrm{eV}$ and $1.3 \times 10^{13} \leq n_{\mathrm{e}} \leq 2.3 \times 10^{13} \mathrm{~cm}^{-3}$ are the relevant plasma parameters in the radial region of interest. Therefore, the electron heat propagation from the LFS to the HFS is of a collisionless nature, associated with the motion of particles under the influence of an electric field $E_{\|}$that is caused by the different behaviours of electrons and ions. Since the electrons acquire a near Boltzmann distribution rather quickly, the electron pressure gradient caused by the temperature perturbation is balanced by the resulting electric force,

$\partial\left(T_{\mathrm{e}} n_{\mathrm{e}}\right) / \partial l=-e n_{\mathrm{e}} E_{\|}$

The deviation from an exact Boltzmann distribution results from an acceleration of the ions in this electric field towards the LFS

$m_{\mathrm{i}} \frac{\mathrm{d} V_{\|}}{\mathrm{d} t}=e E_{\|}$

which then leads in a quasi-neutral plasma to a net motion of the electrons with the same velocity $V_{\|}$.

From Eqs (1) and (2), we obtain the following estimates for the characteristic values of the velocity $V_{\|}$ and of the time delay $\tau_{\mathrm{D}} \approx L_{\mathrm{c}} / V_{\|}$characterizing the electron heat propagation from the LFS to the HFS:

$\begin{aligned} V_{\|} & \approx \sqrt{\Delta T_{\mathrm{e}} / m_{\mathrm{i}}} \\ \tau_{\mathrm{D}} & \approx \frac{\pi q R}{\sqrt{\Delta T_{\mathrm{e}} / m_{\mathrm{i}}}}\end{aligned}$

Taking the largest observed difference between the $T_{\mathrm{e}}$ perturbations on the HFS and the LFS on the same isobar, and taking $q$ values derived from the standard current profile [7], Eq. (4) shows an increase in $\tau_{D}$ with decreasing $\Delta T_{\mathrm{e}}$, yielding $\tau_{\mathrm{D}}=0.39 \mathrm{~ms}$ at a flux surface radius of $r_{\mathrm{fs}}=24.0 \mathrm{~cm}$ and $\tau_{\mathrm{D}}=0.83 \mathrm{~ms}$ at $r_{\mathrm{fs}}=30.5 \mathrm{~cm}$.

The second approach starts from the assumption that the original heat puff is expelled at the time $t=t_{1}$ across the neutral (X) line of the equation $\varphi=\vartheta$, $r=r_{1}$ [9]. This concept is supported by the TEXTOR density and electron temperature diagnostics [8]. The delay time of the perturbation between the LFS and the HFS isobars depends again on the decay rate of the ion sound waves. From the linearized one dimensional
Vlasov equation, it is shown that the dispersion relation of modes with frequency $\omega$ and parallel wave vector $k_{\|}$is

$$
\sum_{j}\left(e_{j}^{2} / m_{j}\right) \int \mathrm{d} v_{\|}\left(\omega-k_{\|} v_{\|}\right)^{-1} k_{\|} \partial F_{\mathrm{s}} / \partial v_{\|}=0
$$

where the summation is over the particle species and the integrals are along the Landau contour. We expand the propagator in the usual way for sound waves, that is $v_{\|, \mathrm{i}} \ll\left|\omega / k_{\|}\right| \ll v_{\|, \mathrm{e}}$, assume Maxwellian electron and ion backgrounds, and evaluate the Cauchy principal parts and the residues of the integrals accordingly. This yields the approximate solution $\operatorname{Re} \omega= \pm k_{\|} c_{\mathrm{s}}$, where $c_{\mathrm{s}}=\left(T_{\mathrm{e}} / m_{\mathrm{i}}\right)^{1 / 2}$ is the sound speed and

$$
\begin{aligned}
\gamma & =\operatorname{Im} \omega=-\frac{1}{2}\left(\frac{\pi}{2}\right)^{1 / 2}\left[\left(\frac{T_{\mathrm{e}}}{T_{\mathrm{i}}}\right)^{3 / 2} \exp \left(-\frac{T_{\mathrm{e}}}{2 T_{\mathrm{i}}}\right)\right. \\
& \left.+\left(\frac{m_{\mathrm{e}}}{m_{\mathrm{i}}}\right)^{1 / 2}\right]\left|k_{\mathrm{H}}\right| c_{\mathrm{s}}
\end{aligned}
$$

To estimate a characteristic length, we introduce the angular co-ordinates $\phi=0$ or $2 \pi$ and $\theta=\pi$ of the inboard detection position in the equation $\phi-\varphi_{0}$ $=q\left(\theta-\vartheta_{0}\right)$ of a field line crossing the line $\varphi=\vartheta$ with a unit pitch at $\varphi_{0}=\vartheta_{0}$. We obtain

$\vartheta_{0}=q \pi /(q-1) \quad$ or $\quad(q-2) \pi /(q-1)$

and, accordingly, $\phi-\varphi_{0}= \pm q \pi /(q-1)$. The required length is therefore

$L_{\|}=\left[R^{2}\left(\phi-\varphi_{0}\right)^{2}+r^{2}\left(\theta-\vartheta_{0}\right)^{2}\right]^{1 / 2} \sim \pi q R /(q-1)$

Thus, from Eq. (6), the time delay between the signal on the corresponding HFS and LFS isobars should be

$\tau_{\mathrm{D}}(\mathrm{ms}) \sim 0.045\left[A_{\mathrm{i}} / T_{\mathrm{e}}(\mathrm{keV})\right]^{1 / 2} q /(q-1)$

where $A_{\mathrm{1}}$ is the ion atomic mass. Taking $R=1.75 \mathrm{~m}$ and introducing again $T_{\mathrm{e}}=T_{\mathrm{i}}=0.1 \mathrm{keV}$ and $q=2$, we obtain for $\tau_{\mathrm{D}}$ a value of $\tau_{\mathrm{D}}=0.4 \mathrm{~ms}$. Since, closer to the plasma edge, one observes that $T_{\mathrm{e}}<T_{\mathrm{i}}$, a situation that corresponds to a smaller Landau damping rate, we may take $T_{\mathrm{i}}=2 T_{\mathrm{e}}$. Then, again for $T_{\mathrm{e}}=0.1 \mathrm{keV}$ and $q=2$, one obtains $\tau_{\mathrm{D}}=0.8 \mathrm{~ms}$. The use of the exact plasma dispersion function, instead of the approximations that lead to Eq. (7), modifies the results by less than $15 \%$.

The $\tau_{\mathrm{D}}$ values obtained from the above estimates are in acceptable agreement with the observed delay time of the heat pulse propagation, thus supporting our proposed model. 


\section{CONCLUSIONS}

Using an ECE diagnostic array located at a certain toroidal position, the radial propagation of the electron heat pulse following sawtooth crashes was measured simultaneously on both the LFS and the HFS. The heat pulse propagation time was analysed with respect to the corresponding isobars on the LFS and on the HFS, deduced from the original unperturbed $p_{\mathrm{e}}$ profile. The arrival of the heat pulse on the LFS isobar occurred systematically earlier (by up to $1 \mathrm{~ms}$ ) than on the corresponding HFS isobar. The difference in the arrival time increases with the isobar radius. This asymmetry of the local heat pulse propagation was initiated by a hot spot - best observed on the $T_{\mathrm{e}}$ contour plots - releasing the 'high temperature content' on the LFS.

These observations suggest that the primary and predominant radial electron heat transport is localized on the LFS, and that the HFS is only fed subsequently by parallel heat equilibration along the connecting magnetic field lines. An estimate of the time delay $\tau_{D}$ necessary for parallel heat equilibration between the HFS and the LFS is in satisfactory agreement with these experimental results. The proposed explanation of the observed transport pattern, that is the competing participation of both perpendicular and parallel electron heat transport, is also supported by the measured asymmetry in the amplitudes of the heat pulses between LFS (larger amplitude) and HFS (smaller amplitude).

These results call in question the concept of a homogeneous, simultaneous diffusive transport across the magnetic surfaces. Instead, for a quantitative analysis of the electron heat transport coefficients, a three dimensional treatment is required.

\section{ACKNOWLEDGEMENTS}

The authors wish to thank the TEXTOR team for operating the machine and they wish to express their appreciation to $\mathrm{H}$. Kever for helpful suggestions during many discussions. MZT acknowledges the support of the Alexander von Humboldt foundation.

\section{REFERENCES}

[1] TUBBING, B.J.D., et al., Nucl. Fusion 27 (1987) 1843.

[2] JAHNS, G.L., et al., Nucl. Fusion 18 (1978) 609.

[3] LOPES CARDOZO, N.J., et al., Nucl. Fusion 28 (1988) 1173.

[4] FREDRICKSON, E.D., et al,, Phys. Rev. Lett. 65 (1990) 2869.

[5] WAIDMANN, G., et al., in Electron Cyclotron Emission and Electron Cyclotron Resonance Heating (Proc. 7th Int. Workshop Hefei, China, 1989), IAEA, Vienna (1990) 92.

[6] SOLTWISCH, H., Rev. Sci. Instrum. 57 (1986) 1939.

[7] SOLTWISCH, H., Messung der internen MagnetfeldStruktur von Tokamak-Plasmen, Rep. Jül-2339, Forschungszentrum Jülich (1990).

[8] KOSLOWSKI, H.R., KRÄMER-FLECKEN, A., in 1992 International Conference on Plasma Physics (Proc. Conf. Innsbruck, 1992), Vol. 16C, Part I, European Physical Society, Geneva (1992) 383.

[9] ROGISTER, A., et al., in Plasma Physics and Controlled Nuclear Fusion Research 1990 (Proc. 13th Int. Conf. Washington, DC, 1990), Vol. 2, IAEA, Vienna (1991) 231

(Manuscript received 6 October 1992

Final manuscript received 13 April 1993) 